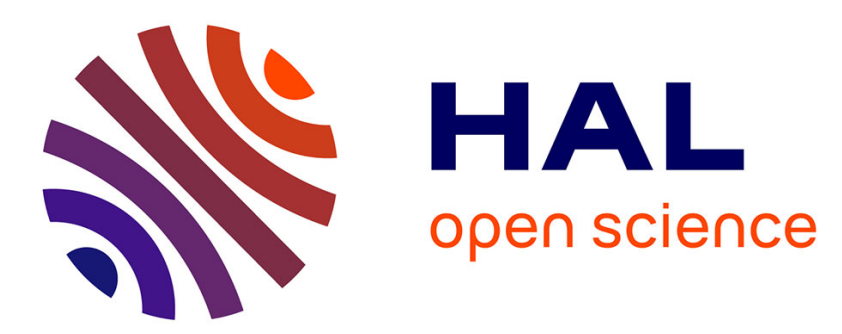

\title{
Paysages de l'hydroélectricité, tourisme et protection de la nature en haute montagne: le Valais suisse
}

\author{
Jean-François Rodriguez., Séraphin Hirtz
}

\section{To cite this version:}

Jean-François Rodriguez., Séraphin Hirtz. Paysages de l'hydroélectricité, tourisme et protection de la nature en haute montagne: le Valais suisse. Projets de paysage: revue scientifique sur la conception et l'aménagement de l'espace, 2014, 10.4000/paysage.11508 . hal-01996148

\section{HAL Id: hal-01996148 \\ https://hal.science/hal-01996148}

Submitted on 28 Jan 2019

HAL is a multi-disciplinary open access archive for the deposit and dissemination of scientific research documents, whether they are published or not. The documents may come from teaching and research institutions in France or abroad, or from public or private research centers.
L'archive ouverte pluridisciplinaire HAL, est destinée au dépôt et à la diffusion de documents scientifiques de niveau recherche, publiés ou non, émanant des établissements d'enseignement et de recherche français ou étrangers, des laboratoires publics ou privés. 


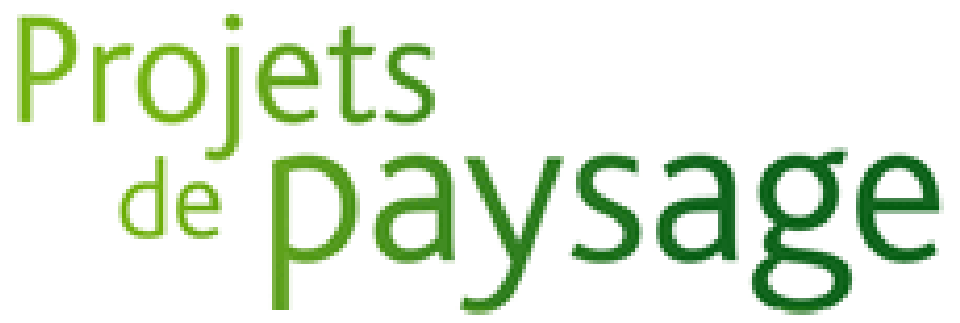

Revue scientifique sur la conception et l'aménagement de l'espace

\section{Jean-François Rodriguez et Séraphin Hirtz}

Paysages de l'hydroélectricité, tourisme et protection de la nature en haute montagne : le Valais suisse

The Landscapes of Hydroelectricity, Tourism and Nature Conservation in a High Mountain Area: The Canton of Valais in Switzerland 
Dans l'histoire du couple paysage - énergie, le paysage de haute montagne et l'exploitation de la ressource hydroélectrique constituent un chapitre majeur. Depuis la découverte de la houille blanche à la fin du XIXe siècle par Aristide Bergès, la vague d'équipements hydroélectriques qui a déferlé dans les montagnes sud-européennes durant le XXe siècle a profondément transformé les paysages. Elle a aussi modifié les structures socioéconomiques valléennes traditionnelles, produisant des mutations territoriales importantes. Outre l'impact paysager des barrages et des infrastructures, le développement extraordinaire du tourisme, favorisé par les aménagements hydroélectriques, est un facteur déterminant de ces changements. Avec le tourisme, le premier constat au niveau territorial est sans doute celui de voir se déplacer et s'élever les limites de la haute montagne, non pas sur un plan environnemental et bioécologique, mais sur celui de l'usage et de la pratique spatiale. En effet, les routes et certains téléphériques construits pour desservir les chantiers hydroélectriques situés en haute altitude ont, une fois les travaux terminés, servi d'accès direct et facile à la haute montagne, sans effort, ni besoin de connaître les techniques spécifiques. Un exemple des plus emblématiques est le parc d'attractions du Châtelard, « fondé en 1975 pour sauver le funiculaire historique ${ }^{1} »$ qui servit à acheminer les matériaux nécessaires aux chantiers des barrages de Barberine (1925) puis d'Émosson (1975) et qui fut emprunté par les touristes dès 1935. Il s'en est ensuivi des transformations des codes des représentations paysagères de la haute montagne. Des regards nouveaux et des normes de valeurs différentes se sont plaqués sur les anciens, faisant émerger de nouvelles formes de patrimonialisation basées sur la dialectique entre le patrimoine issu de l'histoire culturelle et celui mis en valeur par les mesures de protection de la nature (Métailié et Rodriguez, 2011). Aujourd'hui, la majorité de ces installations est toujours en activité pour produire de l'hydroélectricité. Mais certaines, en partie obsolètes et parfois dans un état de ruine avancé, ne répondent plus au besoin de consommation. Par ailleurs, l'attrait exceptionnel de certains sites, du fait de la renommée des installations hydroélectriques et de leur qualité environnementale et paysagère remarquable, a suscité une fréquentation touristique de masse, avec des pratiques spatiales nouvelles, entrânant souvent des dégradations et des problèmes de gestion dans des milieux fragiles. Tout cela confère à ces paysages une apparence dénaturée, voire, selon certains groupes sociaux, une image négative, souillée par l'action et la présence massive de l'homme, alors que les canons esthétiques des « beaux paysages de montagne » revendiquent une nature immaculée, sauvage et préservée. Cette situation radicalise l'opposition entre les partisans de la protection de paysages tenus pour naturels et entre ceux qui intègrent, dans la construction des représentations paysagères, l'intervention de l'homme sur le territoire. Les premiers glorifient une haute montagne vierge de toute emprise humaine pour garantir la beauté des paysages, tandis que les autres prennent en considération ce patrimoine industriel et tiennent compte de son histoire, dans l'idée de construire un projet de territoire cohérent et global. Ils affirment ainsi le caractère hybride 2 des traces sociales dans le paysage montagnard en réfutant l'opposition nature/culture, et placent le facteur anthropique au coeur des réflexions sur les dynamiques paysagères ${ }^{3}$.

À l'heure de la transition énergétique, en vogue dans les débats de société, l'hydroélectricité se présente comme une énergie renouvelable fiable dont on maîtrise la technologie. La 
Suisse, par ses caractéristiques géographiques et sa position centrale en Europe, en fait un atout considérable dans le marché européen de l'énergie. En 2012, un rapport de l'Office fédéral de l'énergie (Ofen) et du Département fédéral de l'environnement, des transports, de l'énergie et de la communication (Detec), basé sur une évaluation des forces motrices suisses en 2007, met en avant le « potentiel latent dans le renouvellement des équipements, la rehausse du niveau de retenue, le dragage, la transformation et l'extension d'installations existantes 4 ». Il propose une stratégie énergétique pour 2050 basée sur des projets de grandes centrales et un développement de la force hydraulique avec des travaux d'envergure. Cette stratégie mise aussi sur de grandes dynamiques paysagères liées aux effets du changement climatique qui laisse prévoir la fonte de la majeure partie des glaciers suisses d'ici 2100 et, de fait, la formation de nombreux lacs glaciaires qui augmenteront de façon considérable les possibilités de la ressource hydraulique. Mais, « le développement de la force hydraulique en Suisse polarise fortement les opinions » avec des préoccupations contemporaines d'ordre écologique, économique et de protection des paysages ${ }^{5}$. Dans un contexte de polémiques entre les « pro-barrage » et les « anti-barrage ${ }^{6}$ », faut-il développer cette production et continuer d'aménager la haute montagne ? Doit-on démanteler les installations obsolètes ? Quel peut être l'avenir de ce patrimoine industriel de haute montagne ? Comment combiner l'inéluctable développement touristique montagnard, porteur du développement d'une économie présentielle ${ }^{7}$ induite par ces aménagements et la protection de l'environnement et des paysages ? En somme, quel sens donner à ces paysages?

Dans la conjoncture énergétique générale d'aujourd'hui, qui englobe une diversité d'enjeux économiques, sociétaux et politiques, les réponses sont complexes. L'histoire de l'aménagement hydroélectrique dans le Valais ${ }^{8}$ témoigne avec acuité de l'enchevêtrement des interrelations entre l'exploitation de la ressource énergétique, la mise en tourisme de la haute montagne et la protection de la nature et des paysages. La lecture du paysage, vu à travers le prisme de l'énergie, met en évidence la complexité de ces liens et révèle l'ambivalence entre l'attirance et le rejet que ces installations provoquent. L'histoire, celle de l'épopée hydroélectrique, présente aujourd'hui des caractéristiques particulières en termes de stratégies d'exploitation énergétique, de développement touristique et d'enjeux de patrimonialisation de l'espace montagnard. Elle met en avant des spécificités importantes, dans la manière de fabriquer les représentations culturelles de ces paysages et dans l'utilisation faite de ces mêmes représentations par les entreprises hydroélectriques, les acteurs de la promotion et du développement touristique ainsi que les organismes investis dans la protection de la nature.

En termes d'exploitation de la ressource et d'aménagement hydroélectrique, le Val d'Hérémence, la vallée du Trient et le Val de Bagnes sont trois vallées majeures, au coeur des Alpes. C'est là que sont concentrés quelques-uns des barrages les plus importants et les plus emblématiques des montagnes sud-européennes, avec respectivement les barrages de la Grande-Dixence, de Cleuson, d'Émosson et de Mauvoisin. Un réseau de connexion gigantesque, composé de galeries souterraines et de conduites forcées, permet la circulation des eaux entre les différentes vallées et alimente un ensemble de centrales hydroélectriques modernes et puissantes, d'une grande capacité de production électrique à l'échelle de la 
Suisse.

\section{L'hydroélectricité support de la mise en tourisme de la haute montagne pour les acteurs « traditionnels » du tourisme}

L'attrait touristique de cette région est, comme pour l'ensemble des Alpes, antérieur à l'avènement de l'hydroélectricité. Au commencement, la mise en tourisme est orchestrée par les acteurs traditionnels de l'économie touristique : hôteliers, voyagistes, offices de tourisme... sont les principaux représentants des métiers traditionnels du tour operating 9 . Cet essor est étroitement lié à la création des clubs alpins ${ }^{10}$ et à la naissance de l'alpinisme à Chamonix, dont la renommée, attachée au mont Blanc, et la relative proximité sont une chance considérable pour nos sites d'étude, en particulier pour la vallée du Trient, qui fait partie de l'Espace Mont-Blanc ${ }^{11}$. Dès son origine, l'exploitation de la ressource hydroélectrique, qui permet l'électrification des vallées, jouera un rôle prépondérant pour le développement touristique. En particulier, cas classique qui se produit dans l'ensemble des vallées touristiques à peu près à la même époque, l'énergie fournie alimente le chemin de fer, comme celui qui « traverse les villages de la vallée du Trient » dès 1908 (Hirtz, 2013). Mais, l'électrification est aussi un avantage qu'ont su exploiter très tôt les hôteliers de ces vallées, pour moderniser leurs établissements et fournir tout le confort recherché par les voyageurs. C'est ainsi qu'en 1926 la Société des hôteliers de Fionnay, dans le Val de Bagnes, fait construire une usine qui « fournit l'électricité aux établissements publics de la station » (Hirtz, 2013). Les touristes allaient y admirer la cascade, elle-même réalisée de toutes pièces en 1890 par ces mêmes hôteliers, en dérivant le torrent au-dessus de Fionnay, dans le but de créer un intérêt pittoresque. 


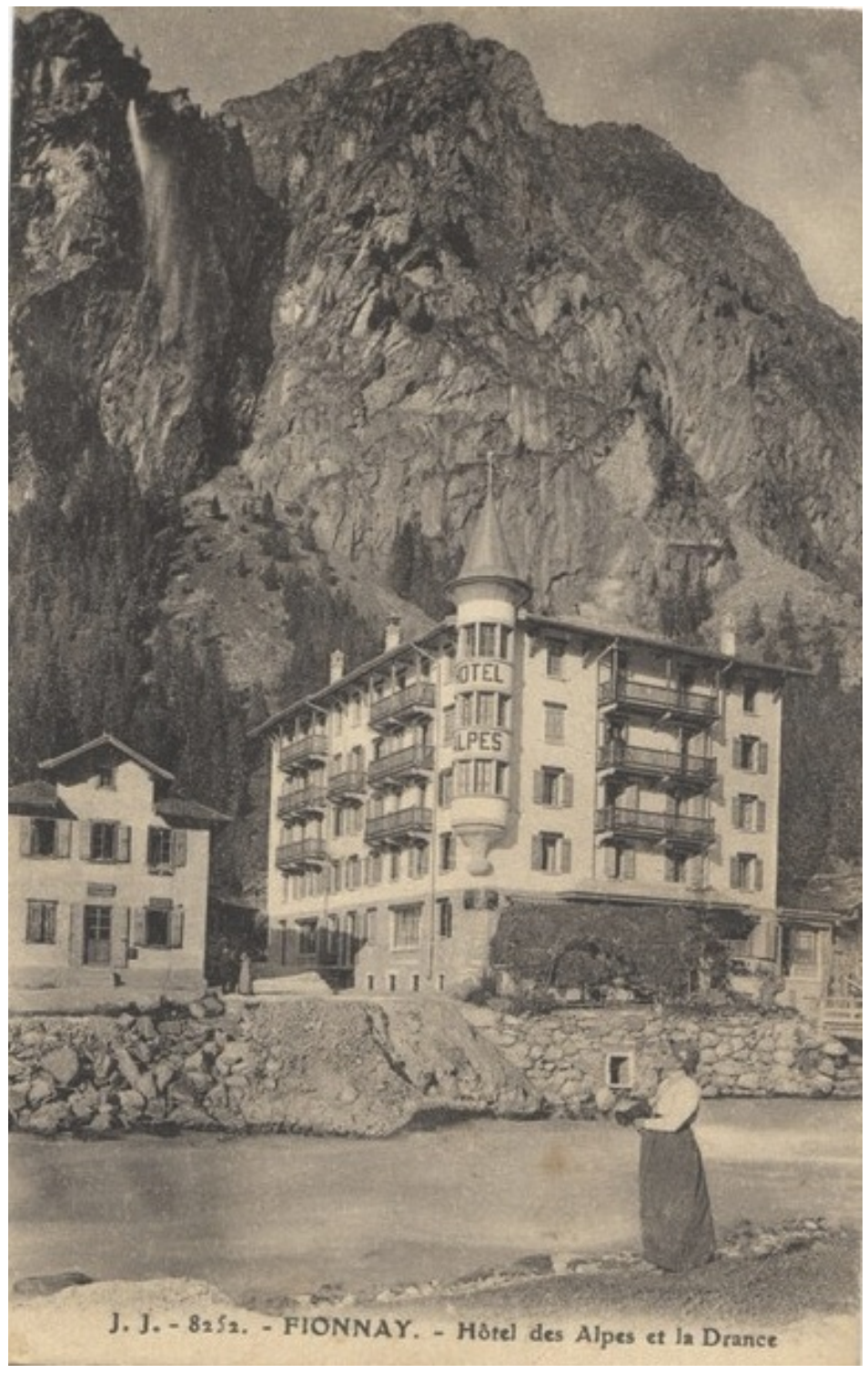

Figure 1. L'hôtel des Alpes à Fionnay, au pied de la cascade créée par les hôteliers, qui jaillit du haut de la falaise (en haut à gauche du cliché). Carte postale des années 1930. Cet hôtel n'existe plus.

À la même époque, dans la vallée du Trient, l'exemple de la construction du barrage de Barberine en 1925, englouti depuis par l'eau du barrage d'Émosson mis en service en 1975, témoigne aussi de la force d'attraction que peut exercer un tel équipement sur la pratique touristique. Le barrage est construit pour accumuler l'eau qui fournira l'énergie nécessaire aux chemins de fer fédéraux. Le lac formé par la retenue, même s'il est artificiel, est très vite investi d'une forte valeur symbolique qui le fait appartenir à la famille des figures paysagères ${ }^{12}$ emblématiques de la haute montagne, au même titre que le pic, la cascade ou 
les séracs des glaciers. Il est très vite devenu « un lieu de loisir fortement prisé par les touristes de Finhaut et Salvan. La commune de Salvan y installe une cabane-restaurant et réutilise les infrastructures de transport ${ }^{13}$ installées pour la construction du barrage » (Hirtz, 2013). Celles-ci, ouvertes au public dès 1935, permettaient aux visiteurs d'accéder facilement au lac de Barberine, où l'on pouvait faire des promenades en barque dans un environnement entouré de hautes cimes et de glaciers, archétype des paysages de haute montagne.

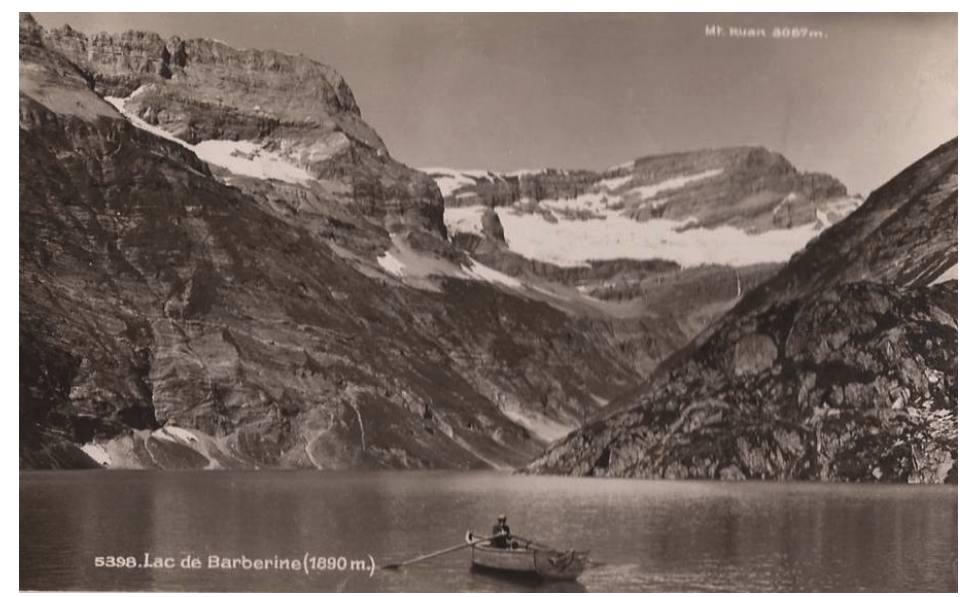

Figure 2. Le lac de Barberine dans son écrin de sommets et les glaciers au fond de la vallée. Touriste faisant une promenade en barque.

Jusqu'à la première moitié du XXe siècle, la mise en tourisme de ces hautes vallées suisses s'appuie sur les infrastructures implantées pour la réalisation des travaux de construction des barrages, leur attribuant surtout une valeur fonctionnelle. La valeur patrimoniale, historique et esthétique est quant à elle donnée aux figures paysagères de référence, suivant des codes de représentation hérités du XVIIIe siècle : les cimes, les glaciers et leurs séracs, les lacs, les cascades, auxquels s'ajouteront rapidement les barrages, emblématiques et empreints d'une forte charge symbolique. Ainsi, à Fionnay, les touristes sont bien attirés par la cascade sortant des falaises au-dessus du village. Peu importe qu'elle ne soit pas naturelle, pourvu que son image le soit. À Barberine, le lac et la possibilité de faire une promenade en barque dans un cadre paysager exceptionnel font l'objet de l'attrait touristique et le petit train qui y mène offre, quant à lui, des paysages panoramiques sur le massif du Mont-Blanc. Le spectacle de la montagne est grandiose sur le site et tout le long du parcours qui y conduit.

Ainsi, fait marquant de cette période, les principaux artisans de ces aménagements sont les protagonistes de l'économie touristique locale traditionnelle, associés aux exploitants de la ressource énergétique. Les hôteliers et les communes investissent en misant sur les potentialités et l'attrait que portent en eux tous ces équipements hydroélectriques.

La nature des liens dans le Valais suisse, entre l'exploitation de la ressource énergétique et 
la mise en tourisme de la haute montagne, présente aujourd'hui bien des similitudes avec le passé. Le tourisme s'appuie sur les ouvrages réalisés à l'époque des grands travaux d'aménagements hydroélectriques entre les années 1950 et 1975. Les barrages de la Grande-Dixence, de Mauvoisin, d'Émosson, sont, avec les infrastructures qui restent de l'époque de leurs chantiers, au coeur du processus énergétique, mais aussi économique de ces vallées. Les cartes postales de cette époque témoignent de la valeur touristique attribuée très rapidement à ces équipements. Là encore, les acteurs dits « traditionnels » du développement touristique se servent de leur image et de leur impact sur la société, en leur attribuant une valeur patrimoniale, au même titre que celle donnée, sur la carte postale, au patrimoine culturel du village de Fionnay et du hameau de Bonatchiesse, situés dans la même vallée.

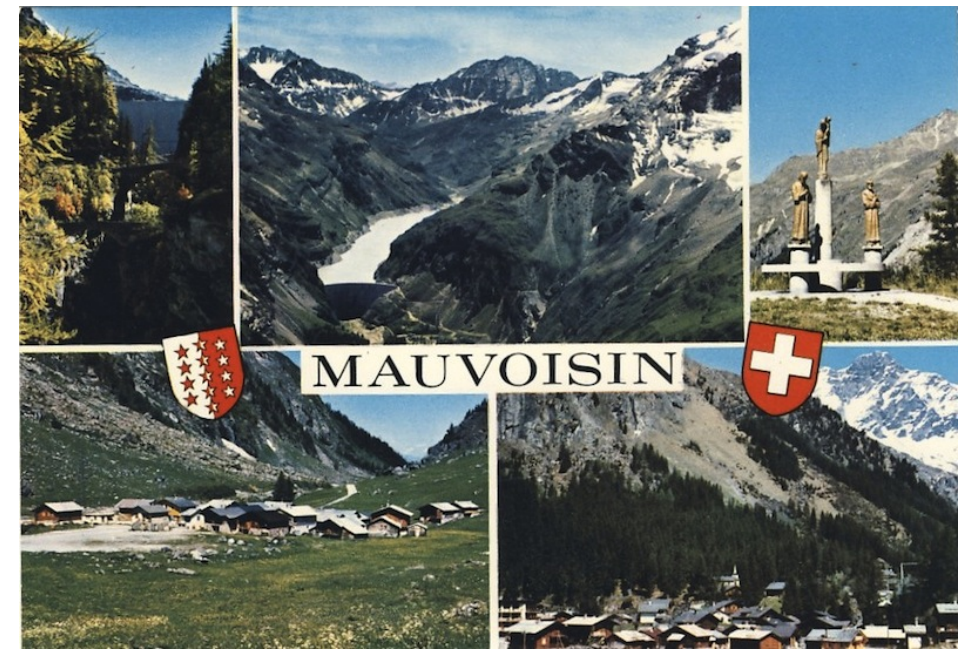

Figure 3. Le nom de Mauvoisin trône au centre de la carte postale, flanqué des blasons du Valais et de la Suisse. Autour, un éventail du patrimoine culturel du Val de Bagnes, donnant autant d'importance au barrage, au lac et au patrimoine datant de cette époque, qu'à l'image d'un patrimoine traditionnel, avec le hameau de Bonatchiesse et Fionnay.

Une des formes les plus emblématiques du développement du tourisme de haute montagne, s'appuyant sur les infrastructures hydroélectriques, est le Tour des Grands Barrages, proposé par l'association de sports de montagne Alptrekking. 


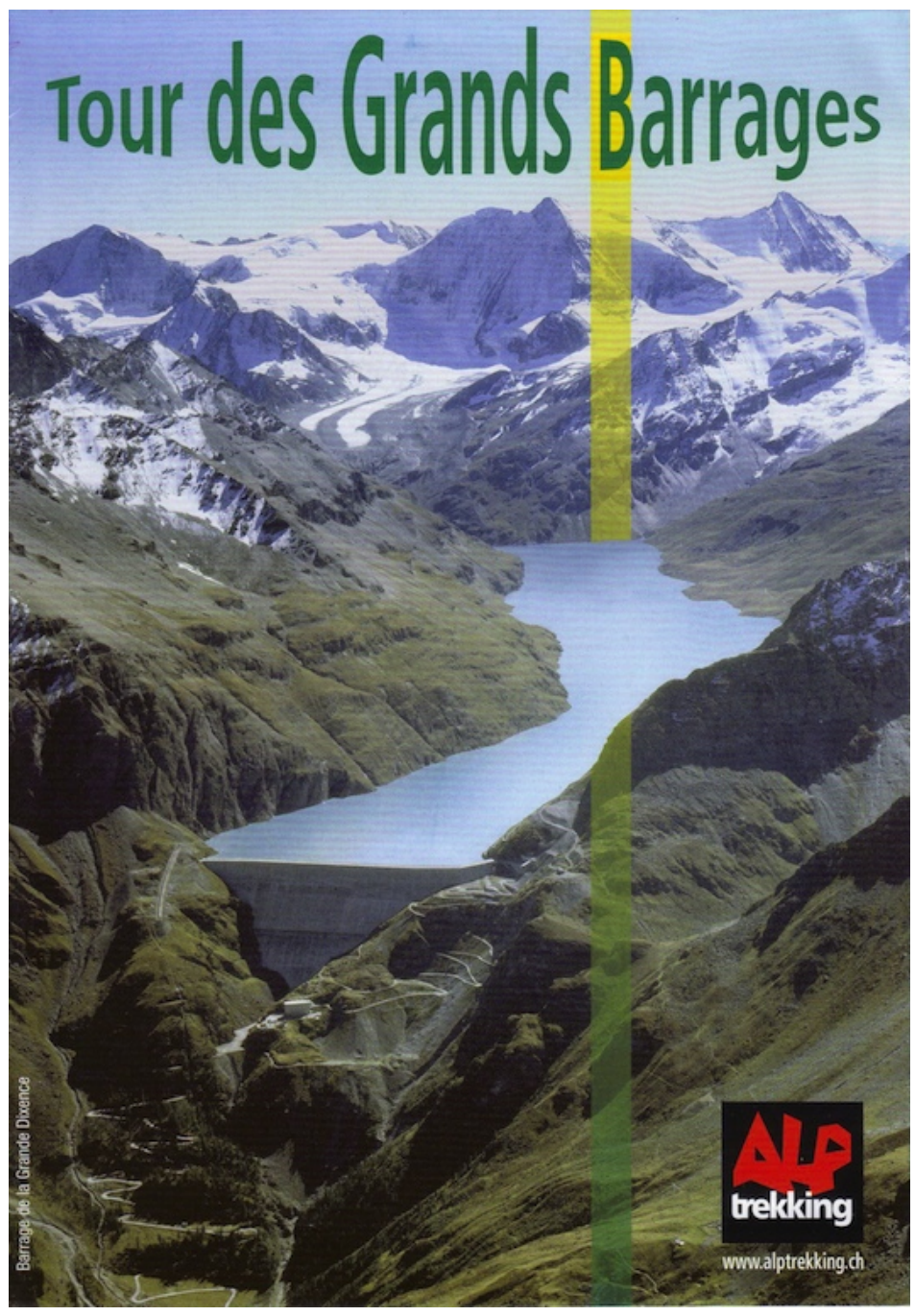

Figure 4. Page de couverture du dépliant publicitaire pour le Tour des Grands Barrages, avec la Grande Dixence, «le plus grand barrage poids du monde».

Celle-ci propose un trek, qui visite les importants barrages de Mauvoisin, de Cleuson, de la Grande-Dixence, de Place-Moulin, dans un tour « qui a comme cadre les plus prestigieux 4 000 des Alpes » et souligne l'intérêt de ce produit touristique pour le développement du tourisme dans les vallées traversées 14 . En accordant ce sens aux infrastructures hydroélectriques et à leur cadre paysager, Alptrekking construit une valeur patrimoniale qui projette simultanément ces figures paysagères emblématiques au coeur du « processus de marchandisation du paysage qui est une des caractéristiques des projets de développement touristiques contemporains » (Chomarat-Ruiz, 2011). Ce type d'action va dans le même sens que celles menées par les organismes institutionnels de la promotion touristique, à l'image de Vallée du Trient Tourisme SA, créée par un groupe de communes de la vallée du Trient pour en promouvoir les intérêts touristiques ${ }^{15}$. Sa brochure générale fait l'éloge des 
paysages grandioses de haute montagne, à la « nature préservée » et « sauvage », portés par la renommée des panoramas «époustouflants» sur le mont Blanc. Dans le chapitre destiné à La vallée du patrimoine, « le site exceptionnel d'Émosson avec ses barrages » est mis en valeur au même titre que les traditions rurales d'origine. Ainsi est établie une stratégie de développement touristique, dans laquelle le patrimoine hydroélectrique du XXe siècle présente le même intérêt que le patrimoine culturel «traditionnel » ou le patrimoine naturel. Tout cela dans une démarche de patrimonialisation qui associe le pittoresque du « charme alpin typique », la modernité et le caractère spectaculaire et sublime des ouvrages hydroélectriques.

\section{Les entreprises hydroélectriques : les nouveaux acteurs du développement touristique de la haute montagne}

À ce processus de mise en tourisme et de patrimonialisation de la haute montagne, conduit par les acteurs «traditionnels » du développement touristique, se sont ajoutés, dans le courant du XXe siècle, de nouveaux acteurs. Les entreprises hydroélectriques elles-mêmes ont contribué volontairement au développement du tourisme, avec la construction de nouvelles représentations culturelles du patrimoine hydroélectrique et la valorisation des paysages de l'énergie.

\section{Mauvoisin : attrait touristique versus valeur patrimoniale de l'histoire}

Dans le site de Mauvoisin, la représentation culturelle se fonde sur la mémoire de l'histoire de l'épopée hydroélectrique et sur l'hommage rendu aux ouvriers de cette aventure humaine. Ici, tout repose sur une mise en scène du paysage de l'hydroélectricité, faite par les Forces motrices de Mauvoisin. Cette mise en scène s'appuie sur l'histoire, tout au long d'un parcours qui mène le visiteur du parking en contrebas du barrage, jusqu'au barrage lui-même. La visite commence par un « chemin de croix », jalonné de bornes en béton, dont le profil évoque celui de la voûte du barrage, toujours présent physiquement dans le paysage. En même temps qu'elles guident le visiteur, comme des cairns le long du chemin, ces bornes ont à la fois un rôle symbolique, de part leur forme et leur matérialité, et une fonction de présentoir des photos des travaux. 


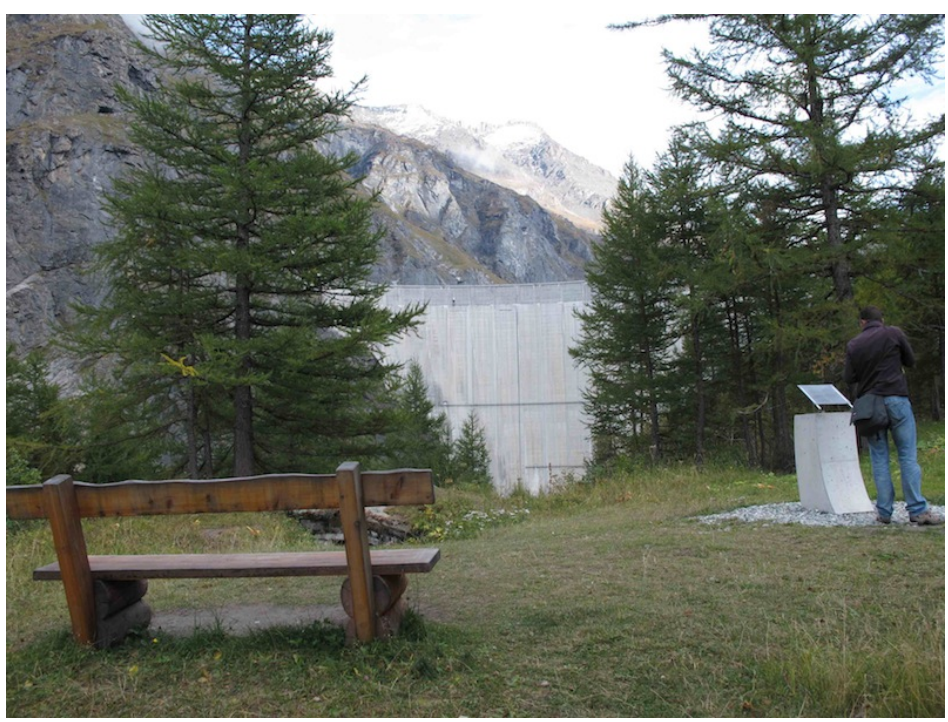

Figure 5. Mauvoisin. Mise en scène du paysage de l'hydroélectricité. Les bornes en béton, rappelant le profil en voûte du barrage, balisent le chemin vers le barrage, comme des cairns. Un banc, placé face à une fenêtre dans la forêt, permet de contempler le paysage du barrage et de la haute montagne dans le fond.

(C) Photo Jean-François Rodriguez

Après ce «premier acte » de la mise en scène du paysage de l'énergie, le visiteur pénètre dans une galerie souterraine, creusée dans la roche, par la porte qu'empruntaient les ouvriers de l'époque. De cette manière, il est «mis dans la peau » de l'ouvrier. Tout en étant spectateur, en regardant les photos en grand format qui exaltent les travaux, il devient aussi, par l'artifice de cette mise en situation, acteur, avec la possibilité de ressentir l'ambiance et les conditions de travail des ouvriers, dans l'obscurité, le froid et l'humidité. 


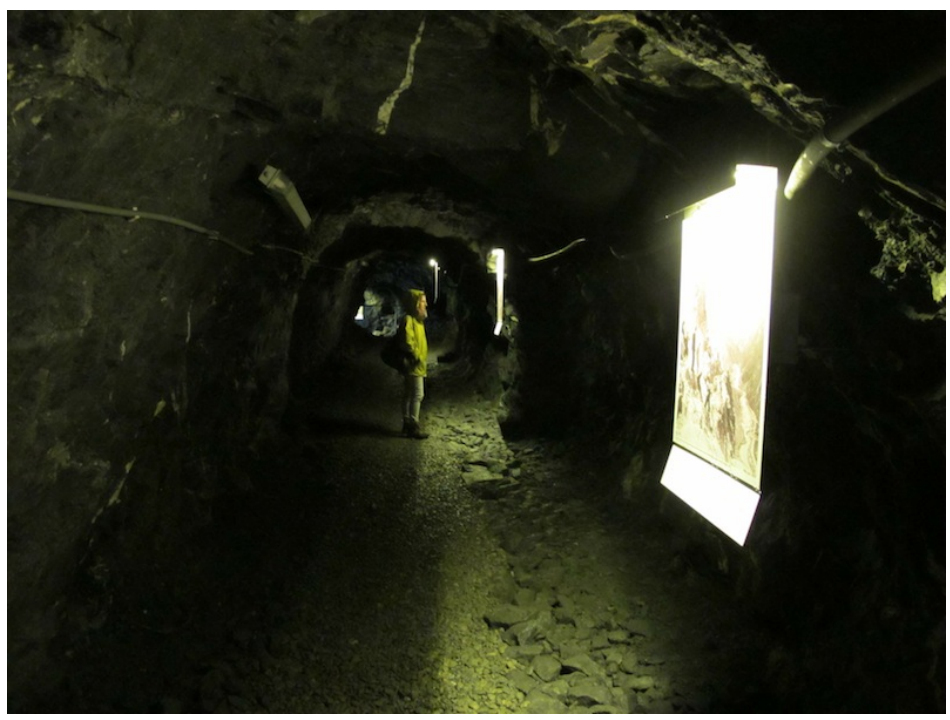

Figure 6. Dans les galeries creusées dans la roche, le visiteur devient acteur, avec la possibilité de ressentir l'ambiance et les conditions de travail des ouvriers, dans l'obscurité, le froid et l'humidité.

(C) Photo Jean-François Rodriguez

Toute cette scénographie s'achève avec l'arrivée sur le barrage, à la lumière naturelle, où le visiteur redevient spectateur d'un paysage éblouissant et grandiose, avec la vue d'un côté sur le bas de la vallée et de l'autre sur les sommets et le lac, dont la couleur émeraude de l'eau fait écho à la pureté de l'eau de fonte des glaciers qui le remplit.

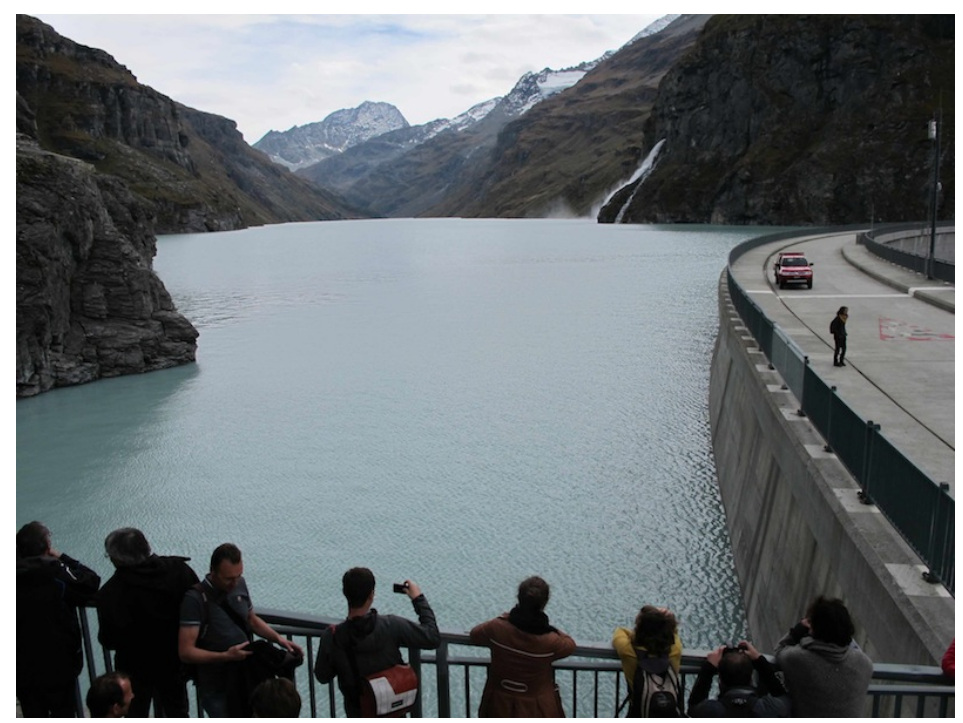

Figure 7. Barrage et lac de Mauvoisin. Le visiteur est mis en situation de spectateur face 
au paysage, dont sommets et glaciers forment le «fond de scène».

(C) Photo Jean-François Rodriguez.

Sur le barrage, la mémoire des ouvriers est glorifiée avec une sculpture gigantesque, à la mesure de l'échelle du site qui révèle l'appartenance de ce patrimoine à la culture et à l'histoire de l'industrialisation de la Suisse et témoigne de sa valeur sociale.

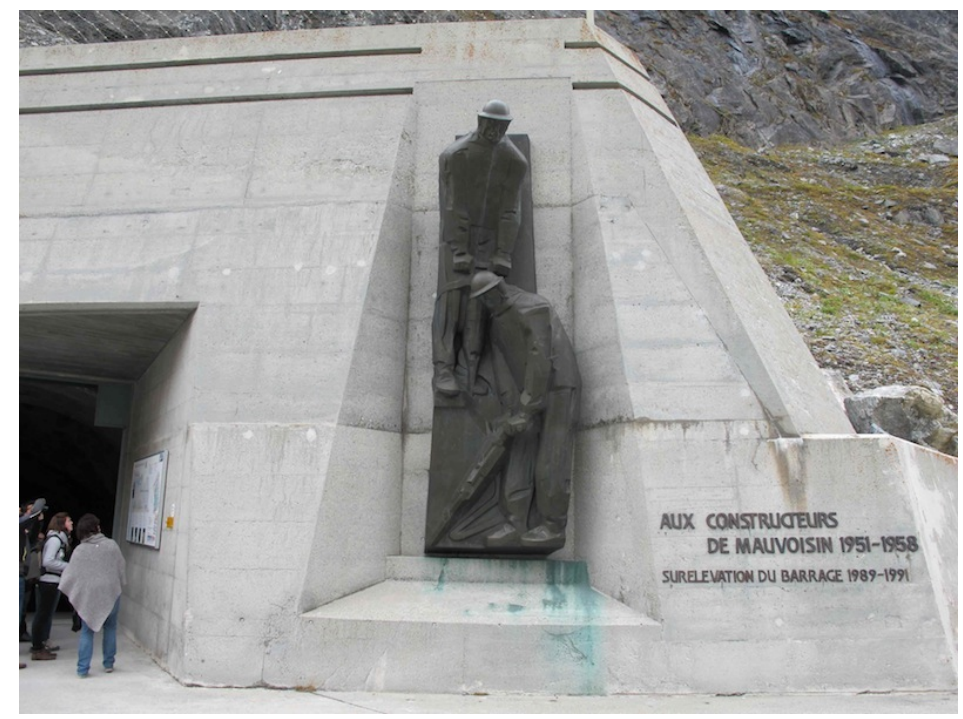

Figure 8. Sur le barrage de Mauvoisin : monument érigé à la mémoire des ouvriers.

(C) Photo Jean-François Rodriguez

\section{Grande-Dixence : attrait touristique versus exaltation de la démesure}

À Grande-Dixence, si les processus de patrimonialisation et de construction de la représentation culturelle du paysage sont aussi fabriqués par le consortium des entreprises qui exploitent la ressource énergétique, la méthode est différente. Les figures paysagères et les éléments patrimoniaux sont les mêmes, la mémoire historique trouve toujours sa place, mais ce sont davantage les superlatifs et les chiffres qui valorisent le site en mettant l'accent sur la démesure et sur le caractère extra-ordinaire des aménagements. Prenons simplement deux passages particulièrement révélateurs dans la brochure touristique distribuée par Dixence SA à la centrale de Bieudron ${ }^{16}$. Le barrage y est présenté, non seulement comme « le barrage-poids le plus haut du monde », mais aussi comme « une légende vivante » :

« À l'échelle des plus hautes montagnes du Valais, cet ouvrage forme un chef-d'oeuvre de technicité et d'audace dédié à l'énergie. Au premier abord on est époustouflé par les $285 \mathrm{~m}$ de béton qui nous dominent mais, une fois sur l'ouvrage, c'est l'émerveillement de la vue du lac des Dix et de la vallée. Le couronnement du barrage forme une gigantesque terrasse panoramique de $15 \mathrm{~m}$ de large sur près de $700 \mathrm{~m}$ de long à $2365 \mathrm{~m}$ d'altitude. » 
«La retenue rassemble les eaux d'un bassin-versant de $420 \mathrm{~km} 2$ recouvert pour moitié par des glaciers. Ce sont ces 35 glaciers qui, au travers de 75 prises d'eau, 5 stations de pompage et $100 \mathrm{~km}$ de galeries, alimentent le lac des Dix ${ }^{17}$.»

Nous pourrions poursuivre avec « les 400 millions de m3 d'eau stockés... Les quelque 2 milliards de $\mathrm{kWh}$ produits chaque année... $18 \gg$. Nous trouvons aussi cet enthousiasme pour la force d'aménagement et la prouesse technique avec le chantier actuel de rehausse du barrage du Vieux Émosson, dont le but est de doubler la capacité de retenue d'eau ${ }^{19}$. Ici aussi, tout comme les visites des barrages et des centrales hydroélectriques, celle du chantier est un attrait touristique considérable. Au-delà du caractère titanesque des aménagements et de l'attitude prométhéenne déployée pour exploiter au maximum des possibilités la ressource en eau, tout cela valorisé en vue d'un intérêt touristique, la première des deux citations ci-dessus nous renseigne aussi sur l'incidence du barrage sur la pratique touristique par son rôle de terrasse panoramique donnant à voir le paysage.

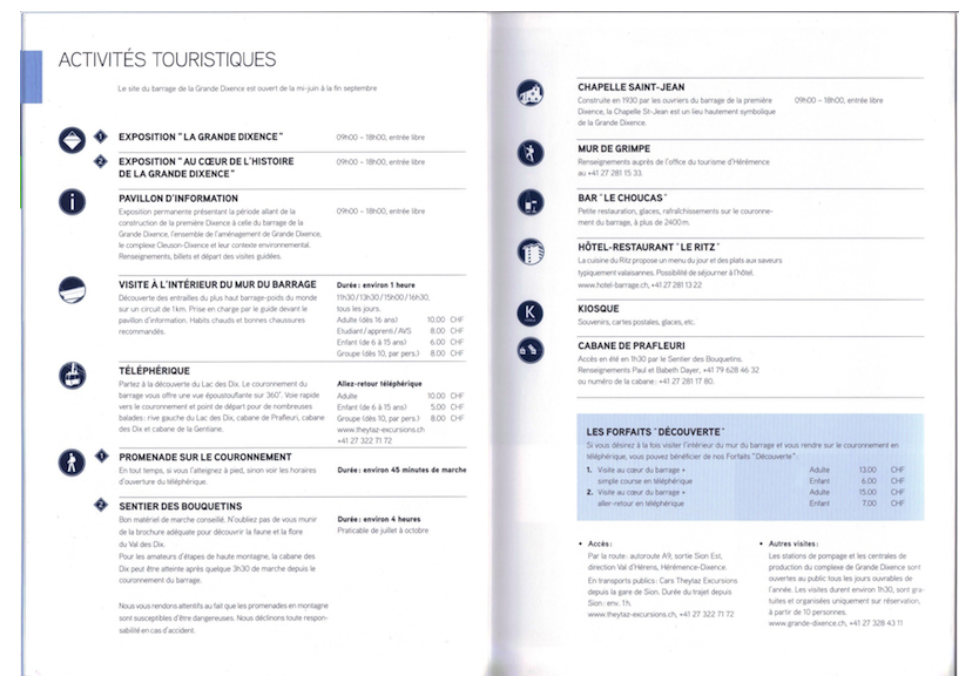




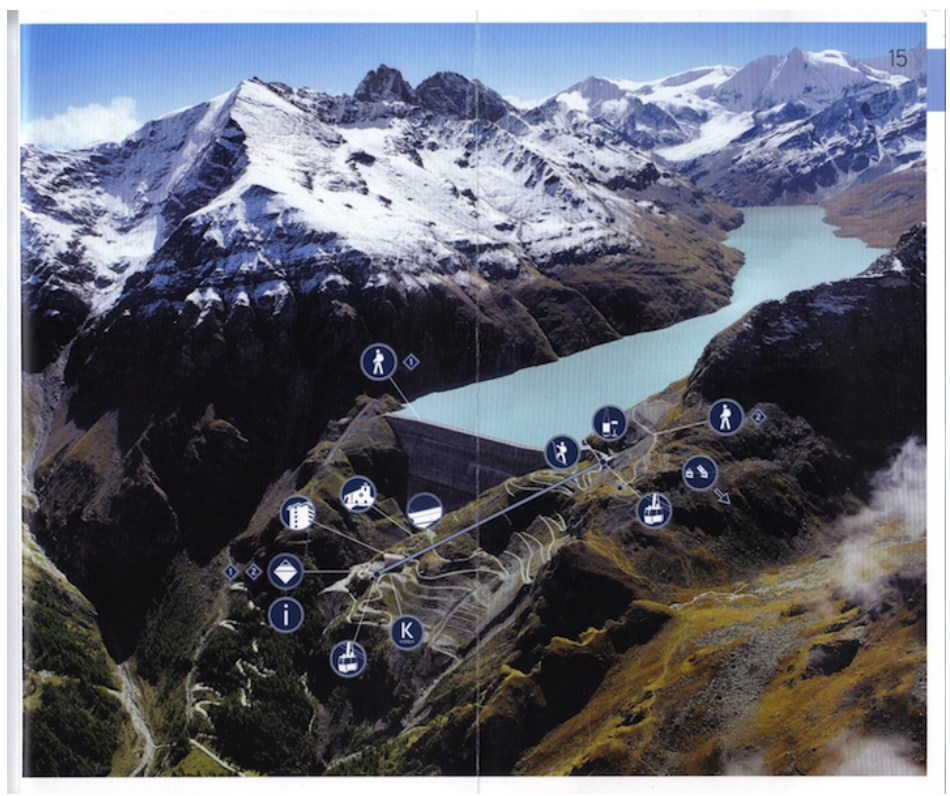

Figures 9. Grande Dixence, Vivre l'énergie au coeur des Alpes. Énumération des activités touristiques autour du barrage, p. 12-15.

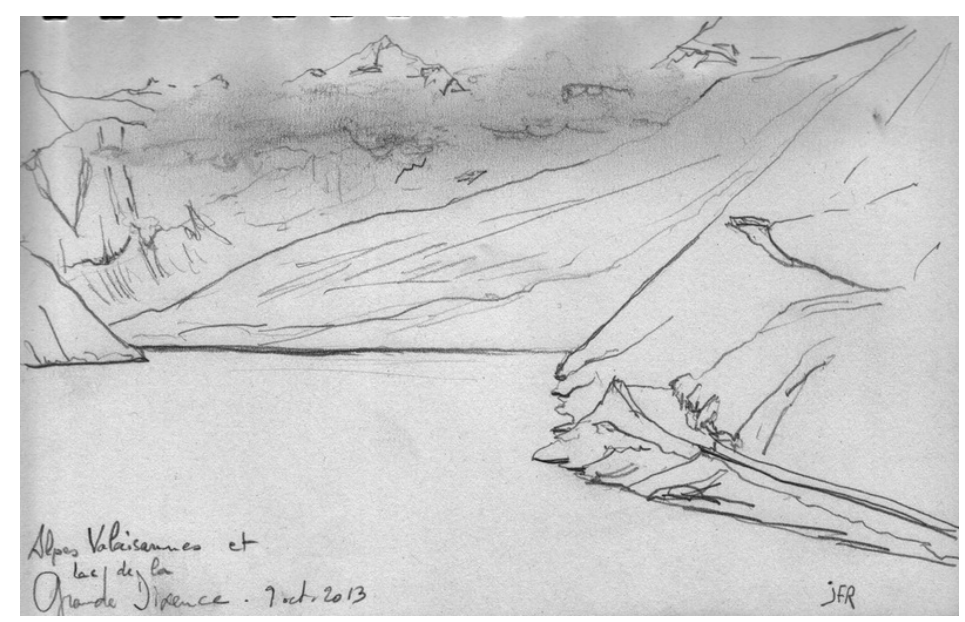

Figure 10. Grande Dixence. Depuis le barrage qui sert de balcon, le visiteur peut contempler le paysage du lac et des Alpes valaisannes.

(c) Dessin Jean-François Rodriguez

Tout comme il attire les visiteurs pour ce qu'il représente d'audace et de technicité dans l'histoire de l'hydroélectricité, il devient le support de contemplation d'un des plus prestigieux paysages de haute montagne du Valais. Le barrage devient ainsi, par effet de réciprocité, « une nouvelle ressource paysagère » (Rodriguez, 2012), à travers les pratiques touristiques qu'il suscite et les nouveaux regards qu'il permet de porter sur le paysage. 
Même si l'exemple du site de Mauvoisin se différencie de celui de Grande Dixence en ce qui concerne leur thématique patrimoniale (effort humain/prouesse technique), ils permettent de comprendre comment la valeur culturelle a contribué de manière significative au développement touristique.

\section{Le tourisme et le nouveau paradigme écologique du paysage de l'hydroélectricité}

Si les aménagements hydroélectriques ont dès le début de leur existence été reconnus et valorisés dans le processus de développement touristique de la montagne, au titre de la technicité, de l'audace et de la modernité qu'ils représentaient, ils ont néanmoins fait l'objet de débats et de fortes réactions. Les préoccupations contemporaines liées à l'écologie et aux contraintes environnementales, associées aux conditions d'acceptation sociale, sont un obstacle majeur au développement de la force hydraulique, dans un contexte suisse d'exploitation des cours d'eau à hauteur de $90 \% 20$.

À partir de la fin des années 1950, face à l'énorme vague d'industrialisation des territoires de haute montagne et au développement touristique qu'elle a généré, la résistance s'est construite en grande partie sur le thème de la protection de la nature et des paysages. Les valaisans ont à ce moment-là pris conscience que « tout le poids du rapide développement économique allait reposer sur le milieu naturel ${ }^{21} »$. C'est donc dans un souci de protection des paysages exceptionnels de montagne, pour faire face aux excès du développement de l'aménagement hydroélectrique, et surtout du tourisme, induit par ces infrasctructures mais aussi par le développement anarchique des stations de ski ${ }^{22}$, qu'a été créée la Ligue valaisanne pour la protection de la nature (LVPN), dans son assemblée constitutive du 21 septembre $1963^{23}$. Quelques années plus tard, le 1er juillet 1966, fut instaurée la loi fédérale sur la protection de la nature et du paysage, dont la première vocation est toujours « de ménager et de protéger l'aspect caractéristique du paysage et des localités, les sites évocateurs du passé, les curiosités naturelles et les monuments du pays ${ }^{24}$ ».

De nos jours, la situation a évolué, avec une dialectique entre développement de l'exploitation et protection de la nature qui implique des compromis au niveau des enjeux des différents groupes d'intérêts dans un nouveau paradigme écologique du paysage de l'hydroélectricité. Dans le cadre de la stratégie énergétique 2050, le Detec considère les critères de développement durable et de protection de l'environnement comme « une référence en matière d'accroissement de l'exploitation de la force hydraulique $25 »$. Dans cette perspective, les entreprises d'exploitation de la ressource hydroélectrique sont tenues de « lier l'aide financière à certaines exigences écologiques 26 » en prenant des mesures compensatoires. 


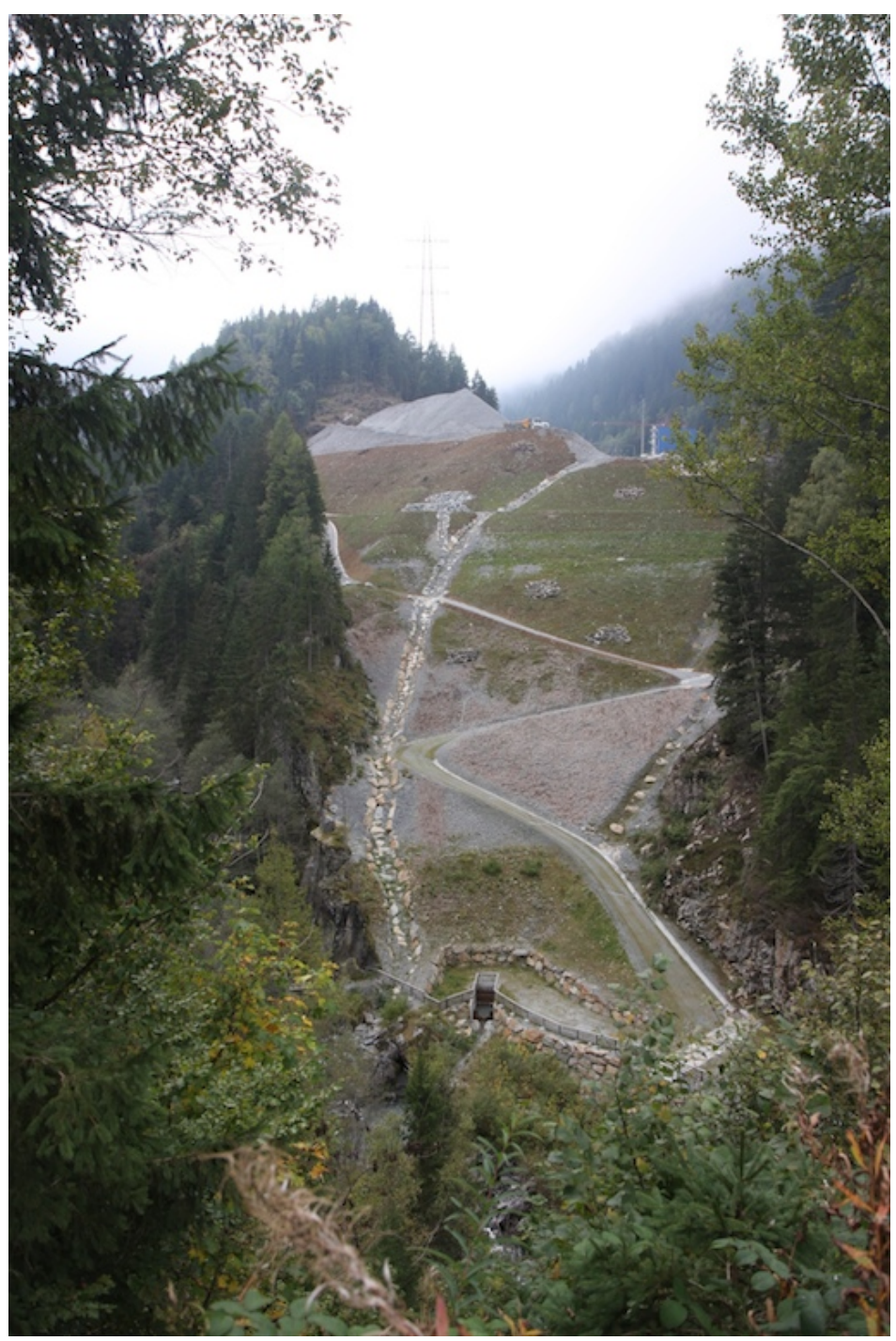

Figure 11. Émosson : travaux de stabilisation du versant en aval du barrage, réalisés dans le cadre des mesures compensatoires écologiques.

(C) Photo Bernard Davasse, 2013

Parfois, elles s'associent avec les organismes de protection de la nature pour préserver l'environnement montagnard, mais aussi pour en révéler sa naturalité dans une démarche de développement touristique. C'est dans ce cadre que « soucieuse de limiter au maximum les effets de ses activités sur l'environnement, Grande Dixence SA a entrepris de développer son propre système de gestion environnementale 27 » et contribué à la création d'une vaste réserve naturelle dans le Val des Dix. Elle a créé, en association avec l'organisme de protection de la nature Pro Natura Valais, le «sentier des bouquetins » : un parcours nature, 
au départ du barrage. Cette action rassemble les différents acteurs autrefois opposés les uns aux autres, dans un projet commun qui vise à maintenir la biodiversité tout en développant une nouvelle forme de tourisme, attirée par le barrage et l'histoire de l'hydroélectricité, les panoramas sur le paysage, la randonnée et l'intérêt pour la flore et la faune.

Aujourd'hui, en Suisse, les acteurs de la production hydroélectrique, ceux des sociétés de négoce de l'énergie et ceux investis dans l'écologie se retrouvent autour de projets communs qui associent l'exploitation de la ressource hydroélectrique, la protection de la nature et des paysages, avec la mise en tourisme de l'espace montagnard. Les entreprises hydroélectriques fabriquent ainsi un nouveau référentiel de représentation des paysages de la ressource énergétique, en les associant à la naturalité, qui devient aussi une ressource, et donnent, par la même occasion, une nouvelle image d'elles-mêmes, en accord avec les préoccupations écologiques et paysagères contemporaines. Elles associent patrimoine culturel, patrimoine naturel et développement touristique, dans un processus d'aménagement du territoire montagnard, respectueux des ressources environnementales et socioculturelles, caractéristique d'un développement durable souhaité par les populations. Ce nouveau jeu d'acteurs contribue à donner une nouvelle valeur patrimoniale aux infrastructures hydroélectriques. Ces dernières s'inscrivent dans un projet de territoire cohérent, avec un développement touristique qui repose sur l'hybridité des traces actuelles dans le paysage en réfutant l'opposition nature/culture. L'évolution de toutes ces interrelations entre l'exploitation de la ressource, la mise en tourisme de la haute montagne et la protection de la nature, ainsi que l'apparition de ces nouveaux jeux d'acteurs ouvrent une nouvelle page de l'histoire des idées et des représentations des paysages de montagne. 
1. www.chatelard.net.

2. Cette hybridité provient du fait que les actions de l'homme sur son environnement combinent les caractéristiques naturelles de celui-ci et les références culturelles de la société, qui elles-mêmes sont imprégnées des relations ambivalentes que l'homme entretient avec la nature.

3. Ce point de vue trouve ses bases théoriques dans la pensée scientifique de Georges Bertrand qui associa l'homme et la nature dans les recherches sur l'environnement et relança les recherches sur le paysage à la fin des années 1970.

4. Le potentiel hydroélectrique de la Suisse. Potentiel de développement de la force hydraulique au titre de la stratégie énergétique 2050, 2012, p. 3.

5. Ibid., chapitre 4, « Réactions », p. 5.

6. Expressions empruntées à Anne Dalmasso, 2008, «Barrages et développement dans les Alpes françaises de l'entre-deux-guerres », Revue de géographie alpine, 2008.

7. L'expression « économie présentielle » est un néologisme issu d'une nouvelle approche de l'économie régionale, qui tient compte du développement de la mobilité, notamment touristique. Définition Insee : «L'économie présentielle regroupe les activités mises en $\AA$ “"uvre localement pour la production de biens et de services visant la satisfaction des besoins de personnes présentes dans la zone, qu'elles soient résidentes ou touristes ».

8. Le terrain d'étude porte sur le Val de Bagnes, la Vallée du Trient et le Val d'Hérémence.

9. Expression anglaise communément utilisée dans le domaine du tourisme pour désigner l'ensemble des entreprises impliquées et des prestations mises en $\AA$ “"uvre dans l'organisation et la commercialisation des séjours touristiques.

10. L'Alpine Club, le premier d'entre eux, est fondé en 1857 à Londres. Le Club alpin suisse et le Club alpin italien en 1863. Le Club alpin français en 1874.

11. L'Espace Mont-Blanc est une initiative de coopération transfrontalière datant des années 1980, et qui réunit 35 communes à cheval sur la Savoie, la Haute-Savoie, la Vallée d'Aoste et le Valais, http://www.espace-mont-blanc.com/.

12. La figure paysagère est constituée d'un élément ou de fragments de paysages qui appartiennent à un système de valeurs associées au paysage, au croisement des représentations sociales et de la réalité matérielle de l'environnement. Elle contribue à la construction de l'archétype du beau paysage de (haute) montagne dans une histoire de longue durée des représentations du paysage. Voir Briffaud, S. « Ressources paysagères et ressources énergétiques dans les montagnes sud-européennes. Histoire, comparaison, expérimentation », 2014 ; en particulier le rapport intermédiaire de recherche, 25 avril 2013.

13. Deux funiculaires et un «petit train panoramique ».

14. http://www.alptrekking.com/.

15. «Créée en janvier 2012 par les communes valaisannes de Vernayaz, Salvan, Finhaut et Trient, la commune française de Vallorcine ainsi que la société de transports TMR », Vallée du Trient Vallorcine. Espace Mont-Blanc (brochure touristique).

16. La centrale de Bieudron, située en bordure du Rhône, est la plus importante des quatre centrales du système hydroélectrique de Grande-Dixence. Elle produit à elle seule une puissance de $1200 \mathrm{MW}$, soit plus de la moitié des $2000 \mathrm{MW}$ de la puissance totale. L'usine se visite. Elle contient une salle d'expositions et de conférences. Deux pratiques qui témoignent du rôle de l'hydroélectricité pour le développement touristique et sa contribution à la production d'un patrimoine culturel. 
17. Grande-Dixence. Vivre l'énergie au cÅ "ur des Alpes, p. 11.

18. Ibid.

19. Dans le cadre du développement de la force hydraulique, au titre de la stratégie énergétique suisse pour 2050.

20. « Le potentiel hydroélectrique de la Suisse. Potentiel de développement de la force hydraulique au titre de la stratégie énergétique $2050 »$, p. 6.

21. http://www.pronatura-vs.ch/. Pro Natura Valais fait partie, en tant que section autonome, de Pro Natura à Ligue suisse pour la protection de la nature.

22. La réserve naturelle du Haut Val de Bagnes fut classée en 1968, suite au développement fulgurant de la station de Verbier (ville-champignon).

23. La LVPN deviendra Pro Natura Valais en 1997, section autonome, de Pro Natura à Ligue suisse pour la protection de la nature.

24. Loi fédérale sur la protection de la nature et du paysage, du 1er juillet 1966 (État le 1er octobre 2013), article 1, 1er alinéa.

25. « Le potentiel hydroélectrique de la Suisse. Potentiel de développement de la force hydraulique au titre de la stratégie énergétique 2050 »,op. cit., p. 18.

26. Ibid., p. 12.

27. Grande-Dixence. Vivre l'énergie au cÅ “ur des Alpes, op. cit., p. 45. 


\section{Jean-François Rodriguez et Séraphin Hirtz}

Jean-François Rodriguez est architecte, enseignant à l'École nationale supérieure d'architecture et de paysage de Bordeaux, membre du Cepage, Centre de recherche sur l'histoire et la culture du paysage, équipe de ADESS UMR 5185 du CNRS/université de Bordeaux.

Courriel : jf.rodriguez@bordeaux.archi.fr

Séraphin Hirtz est architecte paysagiste HES, assistant de recherche à l'Hepia (Haute école du paysage d'ingénierie et d'architecture de Genève) à l'institut Inpact (Institut du paysage, d'architecture, de la construction et du territoire), filière architecture du paysage.

Courriel : seraphin.hirtz@hesge.ch

\section{Bibliographie}

Briffaud, S. (dir.), « Ressources paysagères et ressources énergétiques dans les montagnes sud-européennes. Histoire, comparaison, expérimentation », rapport final de recherche pour l'Atelier international du Grand Paris (AIGP), le Bureau de la recherche architecturale, urbaine et paysagère du ministère de la Culture et de la Communication et la direction de la Recherche et de l'Innovation du ministère de l'Écologie, du Développement durable et de l'Énergie, programme de recherche «Ignis Mutat Res. Penser l'architecture, la ville et les paysages au prisme de l'énergie », 2004, 329 p.

Chomarat-Ruiz, C., «Quelle valeur pour le patrimoine paysager ? Du désir de paysage à la volonté de territoire »,\%u2028 Projets de paysage, publié en juillet 2011,

URL:http://www.projetsdepaysage.fr/fr/quelle valeur pour le patrimoine paysager du desir de paysage a la volonte

Cleuson-Dixence, À la découverte d'un aménagement hydroélectrique unique au monde, brochure touristique, Grande Dixence SA et Alpic, 2010, 43 p.

Dalmasso, A., « Barrages et développement dans les Alpes françaises de l'entre-deux-guerres », Revue de géographie alpine, 96-1/2008, mis en ligne le 3 mars 2009, p. 45-54, URL : http://rga.revues.org/i

Département fédéral de l'Environnement, des Transports, de l'Énergie et de la Communication et l'Office fédéral de l'énergie à division économie, pour le compte de la Confédération suisse, « Le potentiel hydroélectrique de la Suisse. Potentiel de développement de la force hydraulique au titre de la stratégie énergétique $2050 », 2012,24$ p.

Grande Dixence, Vivre l'énergie au $c^{1 / 2 u r}$ des Alpes, brochure touristique, Grande Dixence SA, 2010, 50 p.

Hirtz S., « Séminaire Bagnes/Suisse, Octobre 2013. Lecture énergétique des paysages. Val de Bagnes, Vallée du Trient, Val d'Hérémence, Val d'Anniviers », Hepia, filière architecture du paysage, département CEN, Genève, 165 p. 
Métailié J.-P., Rodriguez J.-F., « Du paysage de la ressource au paysage du ressourcement », dans Antoine, J.-M., Millian, J. (dir.), La Ressource montagne. Entre potentialités et contraintes, Paris, L'Harmattan, 2011, p. 213-230.

Rodriguez, J.F., «Paysages de l'hydroélectricité et développement touristique dans les Pyrénées », Revue de Géographie alpine , 100-2, mis en ligne en décembre 2012, URL : http://rga.revues.org/

Vallée du Trient Vallorcine, Espace Mont-Blanc, brochure touristique, vallée du Trient Tourisme SA., Espace Mont-Blanc, 31 p. 